\title{
Editorial: OJPHI Vol 4, No 2 (2012)
}

\author{
Edward Mensah, PhD $^{1}$ \\ ${ }^{1}$ University of Illinois at Chicago, School of Public Health, Chicago, IL USA
}

Recent World Health Organization data show that while cigarette consumption has been decreasing in the advanced countries over the last decade the worldwide number of smokers is still increasing. The rate of tobacco use has not declined among the youth and young adults in the lower to middle income countries mainly due to aggressive tobacco industry marketing practices. Smoking is a major risk factor for cancer. If the current trend is not curtailed smoking is expected to contribute to 8 million deaths worldwide by 2030. In order to design effective smoking cessation strategies it is important to provide policy makers and epidemiologists with quality and reliable data. Portable handheld computers and electronic data management systems have been used to develop and implement surveys in advanced countries. These mobile health applications have not been widely used in developing countries due to infrastructural and financial constraints.

In a paper titled 'Electronic Data Collection and Management System for Global Adult Tobacco Survey" Pujari, et. al., describe the development and implementation of a Global Adult Tobacco Survey aimed at collecting data to monitor and measure global adult tobacco use. The survey was implemented in 14 countries using an electronic data collection system. The authors demonstrate than an electronic data collection and management system can be used effectively in conducting representative surveys in low- to middle-income countries.

Evidence-based research has demonstrated that obesity increases the risk of health conditions such as coronary heart disease, type 2 diabetes, hypertension, dyslipidemia, stroke, and certain types of cancer. Overweight and obese workers impose financial burden on employers through reduced productivity, absenteeism, and increased health care costs. Effective health promotion program can improve the health of workers and reduce health care costs. In a paper titled 'Metabolic Risk Factor Reduction through a Worksite Health Campaign' Daubert et. al., demonstrate the effectiveness of a web-based, heart-health educational program in reducing risk factors linked to overweight and obesity among workers.

While school-based computer-assisted HIV/AIDS intervention models are known to be effective in improving the HIV knowledge, skills, and attitudes of the youth, such models are ineffective in improving the sexual behaviors of the youth. In a paper titled 'Contextual Mediators influencing the Effectiveness of Behavioural Change Interventions' Angella Musiimenta demonstrated that the incorporation of contextual mediators such as relationship characteristics, familial influences, peer factors, gender-based social norms, economic factors and religious 
beliefs in computer-assisted HIV interventions could improve the sexual behaviours of the youth in Uganda.

An integrated laboratory response system capable of responding to threats in a timely manner is a requirement for effective control of public health emergencies. The Global Laboratory Directory Mapping tool (GlaDMap) is a technology that facilitates connectivity between laboratory networks and thereby makes it easier for the laboratories to provide a coordinated response to pandemics. Mukhi et. al., evaluated the effectiveness of the GlaDMap search tool, the descriptive content of the networks and the profiles of the laboratories (function, location, expertise) in the GlaDMap database. The evaluation revealed a very low participation rate and information sharing in GlaDMap. The authors suggest that the addition of an optional functionality to protect the privacy and security of health information would improve the participation of laboratories in GlaDMap.

There is an Information and Communication Technology (ICT) revolution currently going on in USA and advanced countries. The idea is to deliver quality care cost-effectively, expand access to care, improve care coordination among providers, and address the escalating cost of care. The health ICT revolution is yet to penetrate developing countries due to lack of capital, expertise, and other complementary infrastructures. Emmanuel Achampong reviews the state of ICT investment and adoption in the healthcare sector in Ghana in comparison with other African countries. His research also identifies some of the major constraints to health ICT adoption and makes suggestions for improving the adoption rates.

The Meaningful Use requirements of the Health Information Technology for Economic and Clinical Health Act of 2009 provide public health agencies the opportunity to be integrated into the healthcare sector. To participate effectively in the health information exchanges public health departments must be capable of exchanging data and information among themselves and with other providers. In a paper titled "Quality and Integration of Public Health Information Systems: A Systematic Review" Vest et.al. review the barriers and enablers of effective utilization of public health information systems, focusing on immunization information systems and vital records information systems. The researchers conclude that the problems presented by public health agencies' reliance on a large number of autonomous data providers using different technologies must be addressed in order to improve data sharing among these agencies. An important objective of this journal is to provide a forum for Master's of Public Health Informatics students to share their capstone reports with the general health informatics community. In this issue Osama Chaundhary, a recent MPH graduate of the public health informatics program at the school of public health, the University of Illinois at Chicago, explores the foundations for integrating syndromic surveillance development into the implementation of health information exchange at Yolo County Health Department, California. The report provides useful insights for other financially constrained health departments striving to implement health information exchanges and syndromic surveillance systems. 
Edward Mensah, PhD

Editor-in-Chief

Online Journal of Public Health Informatics

1603 W Taylor St, Rm 757

Chicago. IL. 60612

Email: dehasnem@uic.edu

Office: (312) 996-3001 